\title{
Foam Forming under Dynamic Conditions
}

\author{
Jani Lehmonen, ${ }^{\mathrm{a}, *}$ Elias Retulainen, ${ }^{\mathrm{b}}$ Marko Kraft, ${ }^{\mathrm{c}}$ Jouni Paltakari, ${ }^{\mathrm{d}}$ and \\ Karita Kinnunen-Raudaskoski ${ }^{\mathrm{e}}$
}

\begin{abstract}
Using foam as a carrier fluid in papermaking gives interesting new opportunities. Foam as a more viscous fluid than water is expected to behave differently in a dynamic process. This study presents results obtained under dynamic forming conditions in a semi-pilot scale research environment. Effects of process configurations and running conditions on increased forming speed, web properties, and difference between waterlaid and foam-laid processes are shown. The studies were carried out using a water-laid former and the same environment modified for foam forming. In order to achieve increased forming speed, the open headbox was replaced with a closed headbox, and the former geometry was updated. The process foam was boosted with an additional foam pulper. The foam pulper was used as a machine chest for improving the dispersion of fibers into the foam. A much broader tensile strength ratio range ( 3 to 8$)$ was achieved with foam forming than with water-laid forming. Foam-laid paper had a broader pore size distribution and higher mean pore size. Formation and the formation spectra of foam-laid sheets were more uniform, leading to improvements in the properties of the fiber network.
\end{abstract}

Keywords: Aqueous foam; Foam process; Formation; Forming; Sheet properties

Contact information: a: VTT Technical Research Centre of Finland Ltd, P.O. Box 1603, FI-40101

Jyväskylä, Finland; b: VTT Technical Research Centre of Finland Ltd, P.O. Box 1603, FI-40101 Jyväskylä, Finland; c: VTT Technical Research Centre of Finland Ltd, P.O. Box 1603, FI-40101 Jyväskylä, Finland; d: Aalto University, Department of Bioproducts and Biosystems, FI-02150 Espoo, Finland; e: Paptic Ltd, Tekniikantie 2D, FI-02150, Espoo; *Corresponding author: jani.lehmonen@vtt.fi

\section{INTRODUCTION}

Foam, which has a viscosity much higher than water, enables new opportunities for the paper and board industry. In foam-laid forming technology in paper and board making, an aqueous foam of small spherical air bubbles is used as a process fluid and flowing medium instead of water (Smith and Punton 1975). The foam-laid process can be accomplished using different methods. In this study, a method combining tank generation of foam and a closed headbox was used. The technology is used especially in the nonwoven industry (Hanson 1977), which uses long man-made fibers, mineral fibers, and natural fibers. Processing long fibers is challenging in the water process and, according to Kerekes et al. (1985), increasing fiber length and furnish consistency greatly increase the tendency to form flocs, resulting in non-uniformity of the web. The foam-based process enables the application of long fibers with appropriate formation (Radvan and Gatward 1972; Lehmonen et al. 2013; Kinnunen-Raudaskoski 2017).

Koponen et al. (2016) have reported good formation uniformity from trials with bleached hardwood and softwood kraft pulps and long fibers (6 mm TENCEL ${ }^{\circledR}$ Lyocell fibers). The highest mass fraction of Lyocell fibers was $20 \%$. According to that study, web 
formation with longer fibers improved with decreasing foam density. KinnunenRaudaskoski (2017) has verified the uniform sheet structure using foam forming trials with a 70/30\% share of unrefined softwood pulp and $6 \mathrm{~mm}$ and $12 \mathrm{~mm}$ polypropene (PP) fibers on a pilot paper machine modified for foam technology using a foam density of $370 \mathrm{~kg} / \mathrm{m}^{3}$. The formation was compared with water-formed samples of refined softwood and hardwood pulps, which were tested before the paper machine modification. The forming consistencies were $0.26 \%$ in water forming and $0.61 \%$ in foam forming.

For the paper and board industry, foam-laid forming technology enables new production-scale possibilities to achieve, for example, higher forming consistency without at the same time adversely affecting the formation uniformity (Kidner 1974; Smith and Punton 1975; Lehmonen et al. 2013; Kinnunen-Raudaskoski 2017). The foam-laid process leads to a more porous and bulky structure that influences the strength properties of the sheet (Smith et al. 1974; Punton 1975). However, based on published results, this strength loss can be compensated for by wet pressing of the web or by increasing the refining level of the furnish (Radvan and Gatward 1972; Lehmonen et. al 2019b). Strength properties of foam-formed papers can be modified using micro/nano fibrillated cellulose (Kinnunen et al. 2013; Kinnunen and Hjelt 2016) or polyvinyl alcohol (Kinnunen and Hjelt 2017) as strength additives. In general, the effects of foam forming on paper properties can be explained by the physical effects caused by foam bubbles and partly due to the chemical effects brought about by the applied surfactant (Lehmonen et al. 2019a).

Al-Qararah et al. (2015) have studied the link between fiber network structure and foam properties by comparing pore structure with measured bubble size distribution. Those authors have concluded that foam-formed sheets have larger pores than water-formed sheets, and the pore size distribution is more strongly affected by fiber type than by small changes in bubble size distribution.

The potentiality to expand the raw material base and range of product properties with foam technology enables possibilities to generate new paper and board products. This could lead to the renewal of the current paper and board industry through new and novel product applications.

The research reported here is a continuation of earlier semi-pilot and laboratoryscale studies (Lehmonen et al. 2013, 2019a), which showed certain differences in the viscosity and flow characteristics when foam is used instead of water as a carrier fluid for fibers. These differences are also expected to reflect in the dynamics of water removal, subsequent dry solids content, fiber orientation, structure, and mechanical properties of the web. These are important factors when aiming at upscaling the technology for increased forming speed, improved dewatering, better foam stability, and foam recovery in the forming section.

\section{EXPERIMENTAL}

\section{Materials}

Forming studies were performed using bleached chemical softwood pulp at two refining levels. The Shopper Riegler values were 18 and 23, the average fiber length of the pulps was $2.29 \mathrm{~mm}$, and the fiber coarseness was 167 and $175 \mu \mathrm{g} / \mathrm{m}$, respectively. The fines content (fraction $<0.20 \mathrm{~mm}$ ) was $6.7 \%$ for the lower refining level and $7.4 \%$ for the higher refining level. Tap water was used as the process water. The process foam was produced by tank generation, using sodium dodecyl sulfate (SDS) as a surfactant. The 
average process temperature was $\sim 27^{\circ} \mathrm{C}$ for the foam process. In the tank generation, the air led to the foam is at ambient temperature, and so it partly decreases the overall process temperature. When comparing the foam-laid process to normal water-laid forming process, the process temperature is higher (typically around $50{ }^{\circ} \mathrm{C}$ ) in the water-laid process. Notice that, increased process temperature in the water-laid process decreases viscosity, leading, for example, to improved dewatering properties.

\section{Methods}

\section{Process configurations}

The main principle of the foam-laid forming process is that, instead of water, the raw materials are mixed with process foam in a pulper. In this study, two process configurations were compared for foam. The first process configuration (Fig. 1a.) was further modified in order to increase forming speed, improve dewatering, and to attain better foam stability and foam recovery in the forming section.
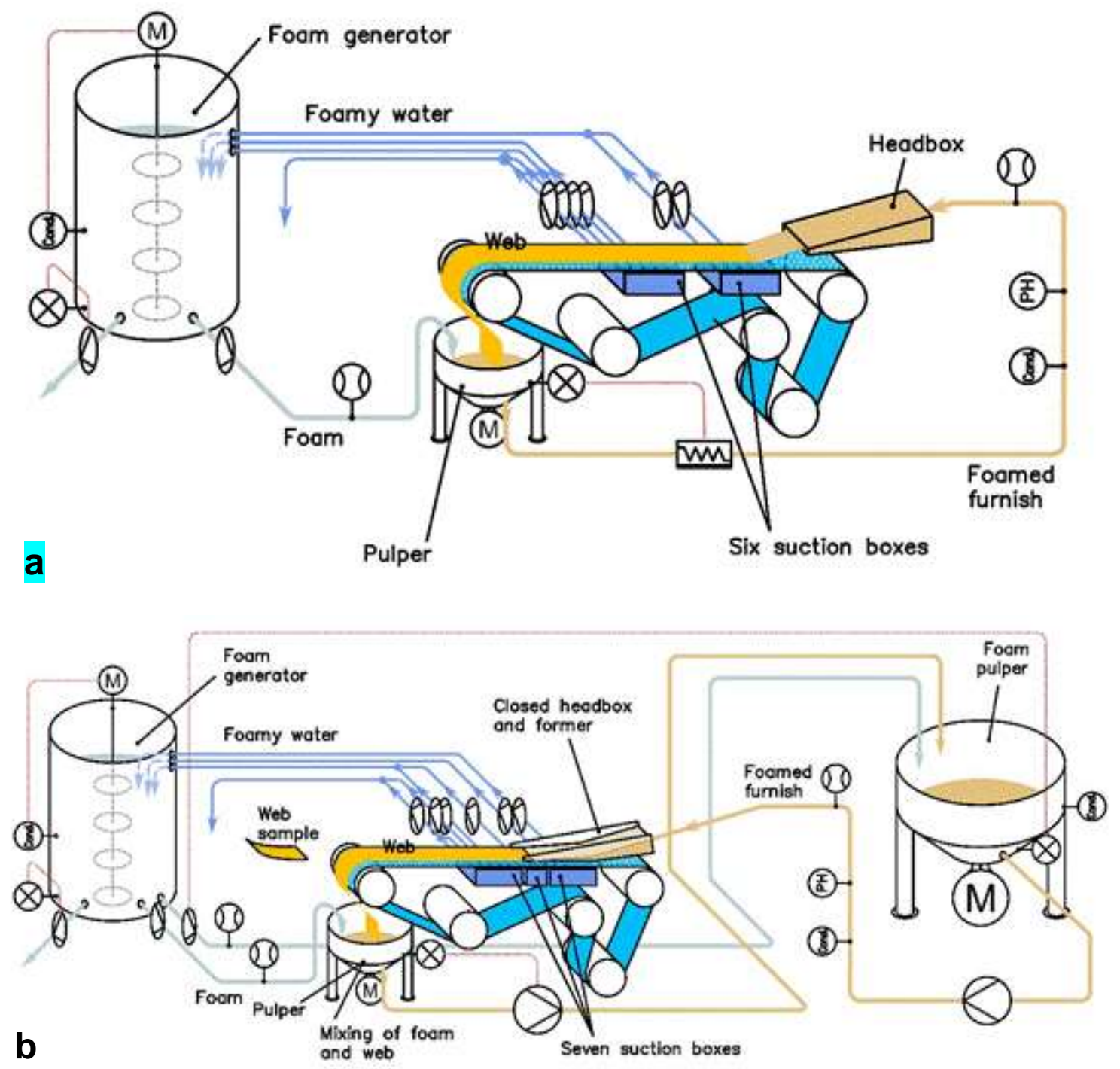

Fig. 1. Schematic diagrams of foam-laid process research environment: a) Open slice-jet forming geometry and foam circulation; b) closed slice-jet based forming geometry and foam circulation 
The latter, modified configuration was also used when making water-laid reference points. A schematic of the research environments before and after the modification is presented in Fig. 1. In this study, the average process foam density in the approach system was $\sim 330 \mathrm{~kg} / \mathrm{m}^{3}$ ( $33 \%$ water in foam), i.e. two thirds of the process foam was composed of air.

The process conditions and formed web properties can be affected by headbox and former parameters, and therefore the major change was related to the forming geometry. The open slice jet based forming geometry, used in our earlier studies, was modified to a closed jet, as shown in Fig. 2. A tapered slice channel forming geometry was used with a $2 \mathrm{~mm}$ slice height.

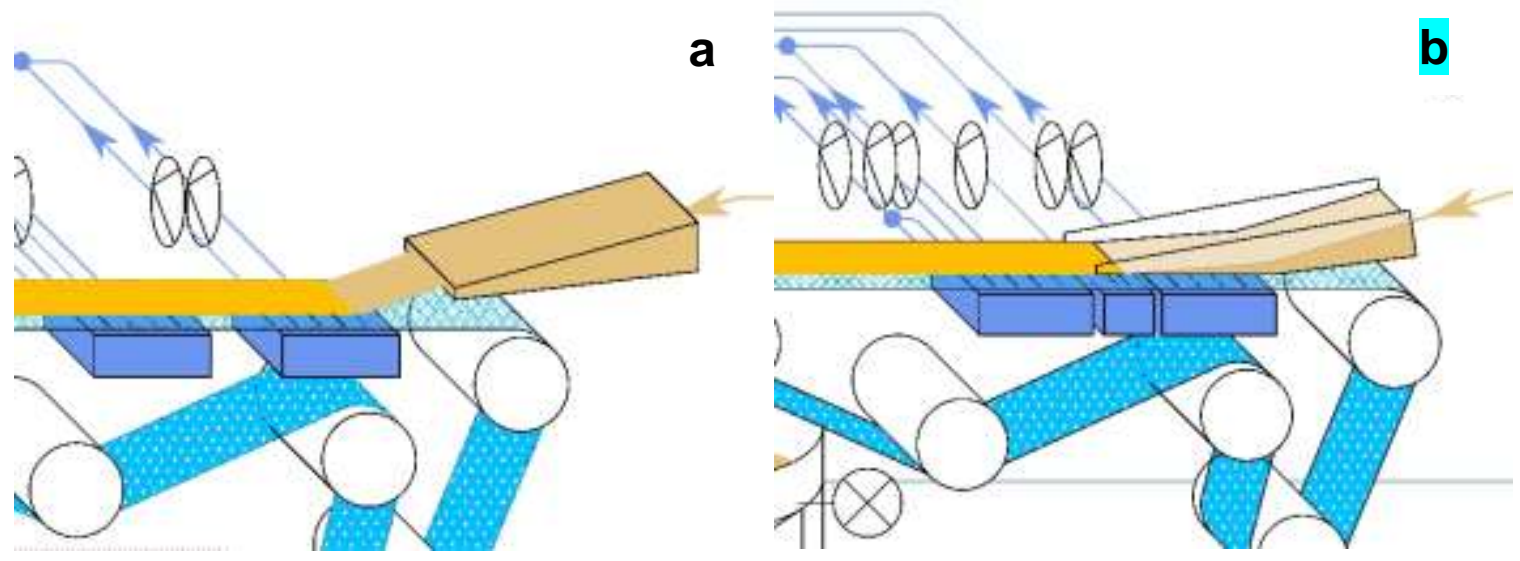

Fig. 2. Open slice-jet and closed slice-jet forming geometries; a) Open slice-jet forming geometry and b) closed slice-jet forming geometry.

By increasing the amount of suction vacuum boxes from six to seven, dewatering conditions were also improved in the forming section. After modification, the first three vacuum boxes were located under the headbox and the former lid, and the additional vacuum box was located just after the slice opening. All of the vacuum boxes could be individually controlled and adjusted without affecting the vacuum levels of the other vacuum boxes. This kind of adjustable vacuum system leads to better process control and foam recovery in the forming section. A removal of the remaining visible foam due to the additional vacuum box is seen as a sharp line on the moving web.
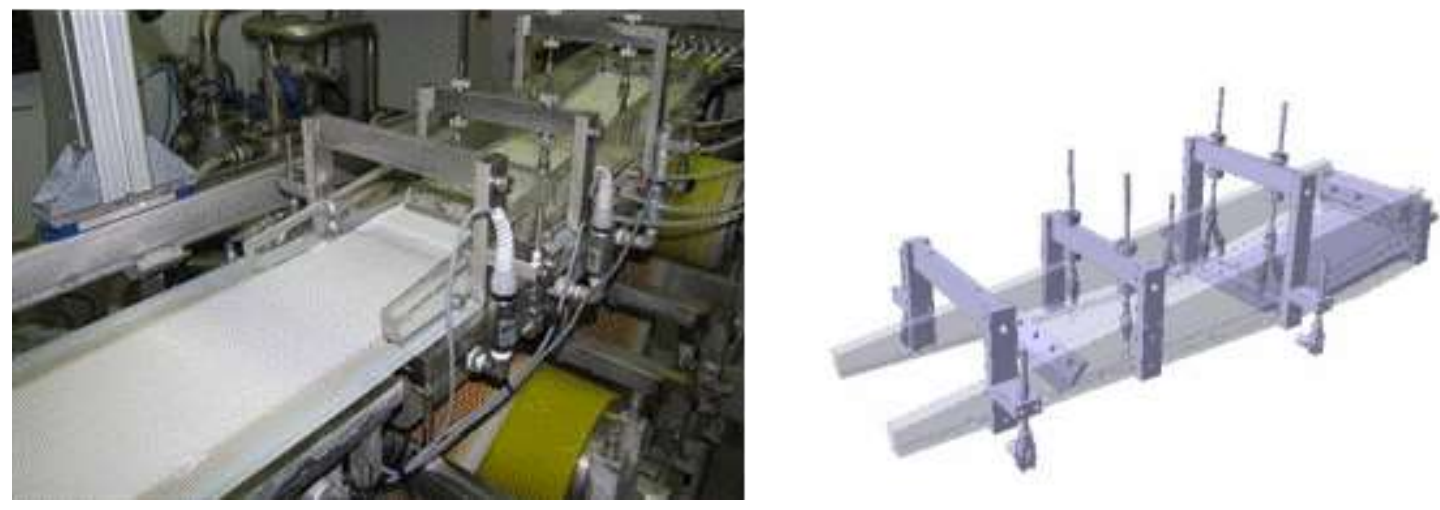

Fig. 3. Closed headbox and former 


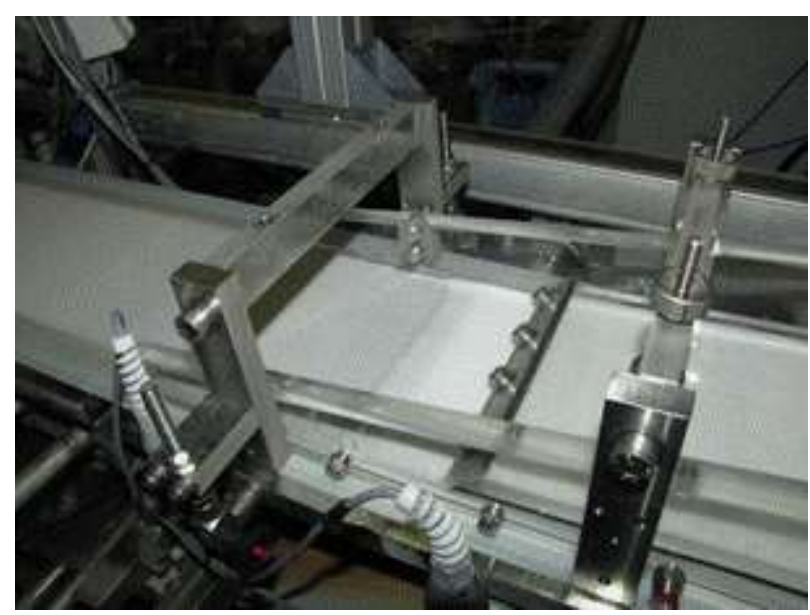

Fig. 4. Sharp foam line after the closed headbox

After the foam line, the amount of process foam in the web is very small and basically, the suction boxes suck air through the web. This air flow is not led to the foam generator and so it doesn't cause any extra air to the process. An example of the sharp foam line is shown in Fig. 4. This headbox solution prevents extra air being led to the process and thus enables a more stable process. More detailed diagrams of the closed headbox and former are shown in Fig. 3.

The vacuum profile used is shown in Fig. 5. Most of the process foam was suctioned and recovered under the closed headbox and the slice opening (suction boxes 1 to 4 ). When the viscous foam had been removed from the formed fiber network, the vacuum levels were dropped to lower levels.

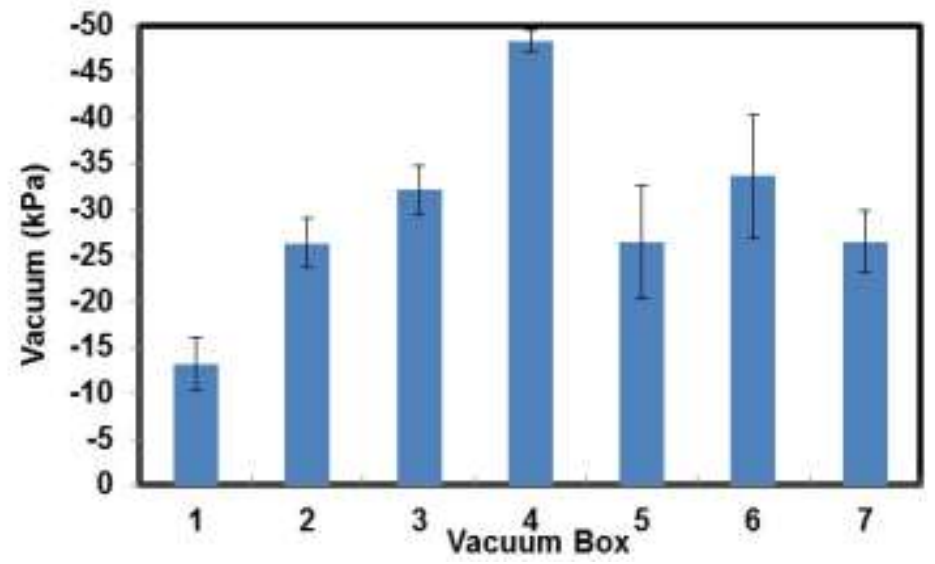

Fig. 5. Vacuum profiles in the forming section. Error bars show the standard deviation for 24 measurements.

The process foam was recirculated within the flow loop. Another process improvement was the installation of a foam pulper (Fig.1b), which was used as a machine chest. The foam pulper enhanced the mixing phase and conditions of the process foam and fibers. Foam produced in the foam generation tank and the dispersed web together with part of the foam in the pulper were pumped into this foam pulper (Fig. 1b). 
A degassing centrifugal pump was used as a feed pump in the approach line. This pump type enables pumping higher amounts of dispersed air-containing foams and suspensions.

When producing process foam, the surface tension of the flowing medium must be modified utilizing different kinds of surface active agents that boost the dispersion of air into the flowing medium and process fluid (Nicolaysen and Borgin 1954; Riddel and Jenkins 1976).

In this study, anionic sodium dodecyl sulfate supplied by Sigma-Aldrich (Darmstadt, Germany) was used as a surface active agent. The dosing system for the surface active agent used for foam generation and control of the quality of the new and recovered process foam was the same as in earlier studies (Lehmonen et al. 2013). The air content of the foam correlates with conductivity measurements (Kruglyakov 1999; Weaire and Hutzler 1999), and this special feature could be utilized, for example, in planning the control system of the foam forming process at production scale. In the present study, the air content of the process foam was followed online based on the conductivity measurement characterized in the foam generator.

In the case of foam, the relevant definition for forming consistency is to calculate based on the dry weight of fibers per total weight of the sample, because the volume is dependent on the air content of the fiber foam suspension and it can be varied over a wide range. The forming consistency was $\sim 0.80 \%$ by weight for water-laid paper and $1.0 \%$ by weight for foam-laid paper.

The target grammage in the trials was $80 \mathrm{~g} / \mathrm{m}^{2}$, and the target foam density was 300 to $330 \mathrm{~kg} / \mathrm{m}^{3}$ (measured at atmospheric pressure in the foam generator). Jet-to-wire ratio series were run from 0.6 to 2.5 in foam forming and, correspondingly, from 0.9 to 1.25 in water forming.

Water-formed paper was produced as the reference in the same research environment by changing the closed headbox to an open slice jet headbox and by changing the foam recovery system to a conventional wire pit system. The width of the web was 160 $\mathrm{mm}$ for foam-laid papers and $140 \mathrm{~mm}$ for water-laid papers.

\section{Raw material characterization}

The pulp properties were characterized using the fiber analyzer Fibre-Master (Lorentzen \& Wettre, Stockholm). The Shopper Riegler value was determined according to ISO standard 5267-1 (1998).

\section{Paper sampling}

The formed paper web was sampled after the forming section without wet pressing, using a sampling apparatus or through simultaneous, gentle wet pressing using a roll nip. Afterwards, unpressed paper samples were wet pressed at wet pressure levels of $50 \mathrm{kPa}$, $150 \mathrm{kPa}$, and $350 \mathrm{kPa}$ according to ISO standard 5269:1 (2005). The unpressed and gentle pressed samples were dried with a cylinder drying device (Kodak rotary drum dryer), and the wet pressed paper samples were dried between a plate and a cloth in a standard airconditioned room at $\mathrm{RH} 50 \%$ and $23{ }^{\circ} \mathrm{C}$.

\section{Paper testing}

All paper samples were stored and analyzed in a standard air-conditioned room at RH $50 \%$ and $23{ }^{\circ} \mathrm{C}$. The grammage of the paper samples was determined according to ISO standard 536 (2012), while the thickness of the samples was determined according to ISO 
standard 534 (2005), and density and bulk were based on the measured values of the grammage and sheet thickness.

The tensile strength properties of 10 parallel paper samples were measured using a Lloyd tensile tester in accordance with ISO standard 5270 (2012) in both the MD and CD direction. In order to equalize the effect of fiber orientation, geometrical average values were calculated (Htun and Fellers 1982). The z-directional tensile strength was measured according to ISO standard 15754 (2009). The scattering coefficient of sheets was measured with a Minolta Spectrophotometer CM-3610d according to ISO standard 9416 (2009).

\section{Formation and fiber orientation}

Based on the $\beta$-formation measurement with Carbon-14 as the radiation force, a storage phosphor screen (SPS) was exposed to $\beta$-radiation through the paper sample. This was done in order to obtain the radiation absorption map. Thereafter, the screen was scanned with a Fuji BAS-1800 II SPS reader. The measured values were converted into a grammage map. The size of the scanned area was $100 \mathrm{~mm} \times 100 \mathrm{~mm}$, and the scanning resolution was $100 \mu \mathrm{m}$. Following this, the resolution was transformed to the Ambertech resolution $(\varnothing 1 \mathrm{~mm})$ and the specific formation value was calculated (Lehmonen et al. 2013).

Tensile strength ratio (MD/CD) was used for estimating the fiber orientation anisotropy of the restrained dried sample sheets.

\section{Bubble size and pore size distribution}

The average bubble size of the process foam in different locations was characterized using a method developed by Lappalainen and Lehmonen (2012). The Sauter mean radius $r[3,2]$ was used for characterizing the bubble size of the process foam.

Based on the article (Lappalainen and Lehmonen 2012) bubble size distributions (BSDs) were characterized using the bubble mean radius $r[1,0]$ and the area-weighted mean radius, i.e. the Sauter mean radius, $r[3,2]$ discovered by the German scientist Sauter (1926):

$$
r[1,0]=\frac{1}{n} \sum_{i=1}^{n} r_{i} \text { and } r[3,2]=\frac{\sum_{i=1}^{n} r_{i}^{3}}{\sum_{i=1}^{n} r_{i}^{2}}
$$

where $r_{i}$ is the radius of the bubble $i(\mu \mathrm{m})$ and $n$ is the total number of bubbles. This is a known method, and several authors have used Sauter mean radius or diameter when describing BSD distribution (Engelsen et al. 2002; Phongikaroon et al. 2006; Junker 2006; Moruzzi and Reali 2010).

The Sauter mean bubble size was measured using the samples from the process. The pure process foam containing samples were taken from the foam generator and headbox.

\section{Pore size distribution}

The pore size distribution of the papers was defined with mercury porosimetry measurement using the method by Koivula et al. (2011). 


\section{RESULTS AND DISCUSSION}

\section{Effect of Process Configuration on the Foam-Laid Process}

Forming speed and foam recovery

The changes in the process configuration made it possible to increase the running speed of the process. The targeted speed enhancement of the process modification was achieved. In the present study, the average forming speed was increased from about 100 $\mathrm{m} / \mathrm{min}$ up to $300 \mathrm{~m} / \mathrm{min}$ with the improved foam-laid process. The installation of a higher capacity headbox feed pump and an increase in the foam removal capacity of the forming section were applied when targeting higher forming speed and foam recovery. In an earlier study (Lehmonen et al. 2013), the maximum forming speed was $125 \mathrm{~m} / \mathrm{min}$, being restricted by stable dewatering and stability of the foam, and formation of the web.

The updated forming geometry enhanced the control of foam recovery. The stability of the process foam was better, as additional air leakage to the vacuum system was prevented. Air leakage decreases the capability of the vacuum system to handle the foam flow and also forms frothy foam. In addition, the closed headbox forming geometry resulted in improvements in dewatering conditions together with an updated vacuum system.

\section{Effect of Forming Methods on the Web}

\section{Formation}

Based on the research of Kerekes et al. (1985) and Martinez et al. (2001), the tendency of the fiber slurry to form flocs and cause a non-uniform web structure is noticeably increased with increasing furnish consistency and fiber length. In this study, the influence of refining level was studied for water forming and foam forming. Specific betaformation and formation spectra were used in evaluating small-scale basis weight variation and how the variation was distributed along different wavelengths. Results for water-laid and foam-laid papers are shown in Fig. 6 and 7.

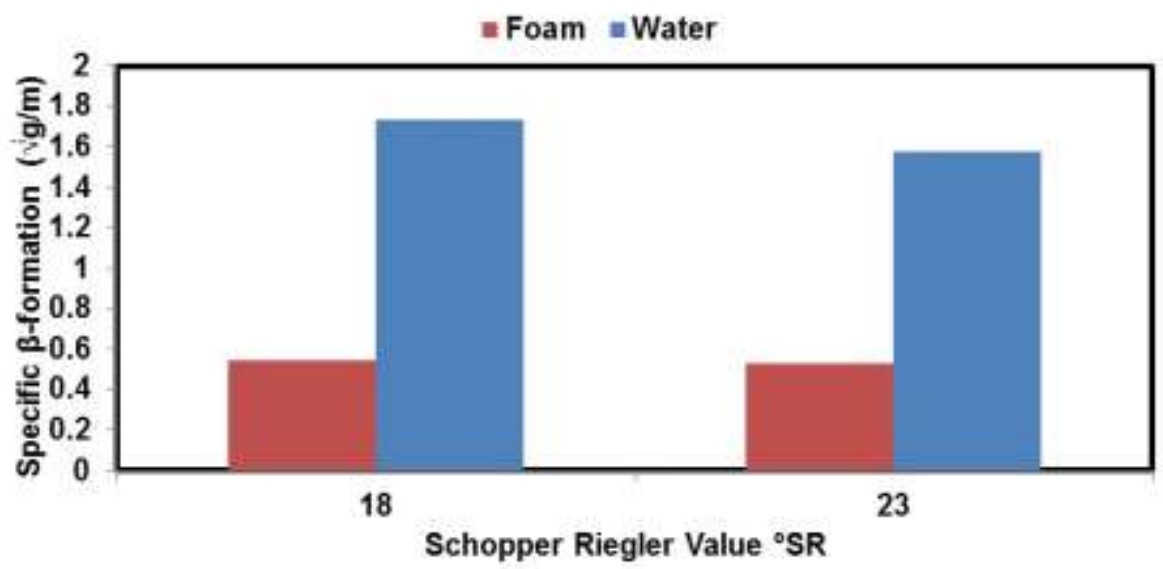

Fig. 6. Specific $\beta$-formation as a function of Shopper Riegler value when closed slice jet forming geometry was used for foam-laid papers and open slice jet forming geometry for water-laid papers

In a water-laid flowing medium, the rheological behavior of fiber suspensions results in fibers flocs, leading to a non-uniform fiber network (Kerekes 2006). This kind of fiber behavior in a water-laid flowing medium induces a deterioration in formation. The 
formation of foam-laid paper was distinctly better than that of water-laid papers, which is in line with the earlier study (Lehmonen et al. 2013). Generally, refining improves formation in water-laid forming, which can be seen as a slightly better formation result (Fig. 6), but did not have any effect on foam-laid forming in this study. This means that in foam-laid papers the refining level could be decreased without weakening the formation.

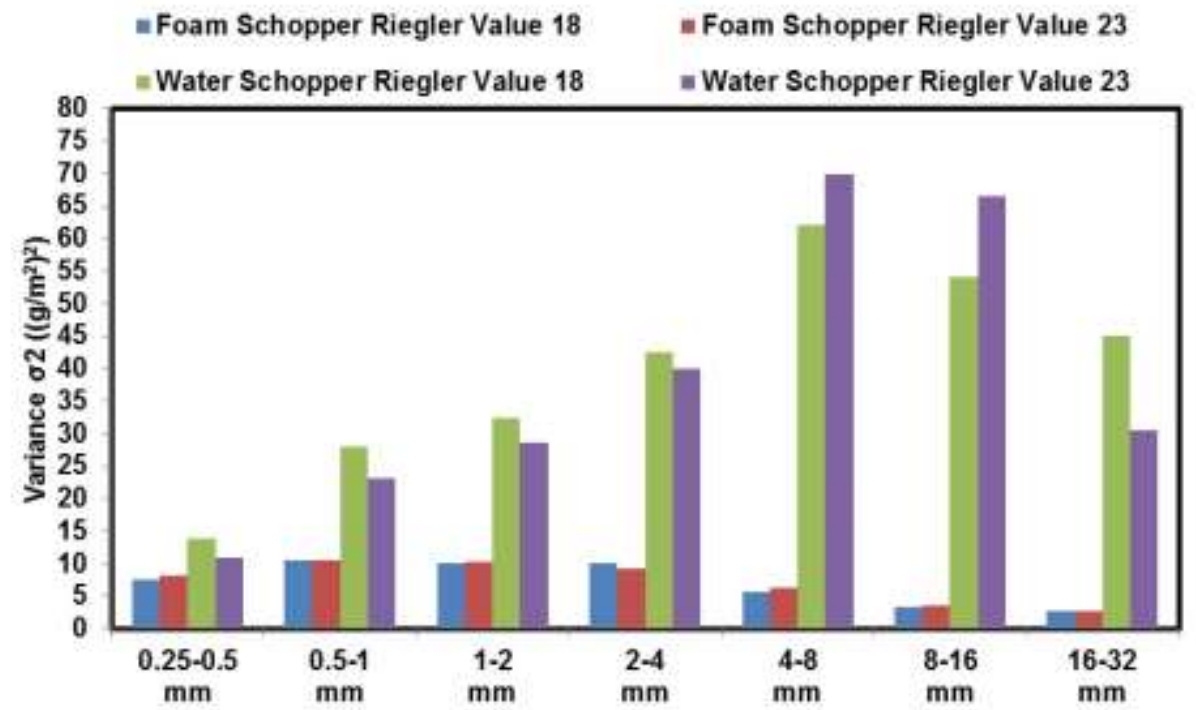

Fig. 7. Effect of forming method and refining degree on the formation spectrum (variance) of basis weight at different wavelengths when closed slice jet forming geometry for used for foam-laid papers and open slice jet forming geometry for water-laid papers

In addition to improved formation, the formation spectra of foam-laid sheets were better in all wavelengths, and especially large improvements were obtained in large-scale variance for both refining levels (Fig. 7). Furthermore, when comparing the formation spectra between the open and closed foam forming geometries, the formation spectra (Fig. 8) for the closed geometry were even better in all wavelengths. The enhancements in process scale, especially mixing conditions and forming geometry, led to improved formation. According to the earlier findings for formation, the variance behavior of the foam-laid sheets was smaller compared to water-laid sheets as a function of wavelength because foam bubbles between the fibers effectively prevent the formation of fiber flocs in a fiber network (Lehmonen et al. 2013; Lehmonen et al. 2019a). According to Kerekes (2006), fibers have a tendency to form fiber flocs in water-laid flowing medium based fiber networks. The benefits of foam bubbles can be seen across the whole spectrum area, but especially in the wavelength area of 4 to $16 \mathrm{~mm}$.

\section{Density}

The density of the formed sheets as a function of wet pressing level is presented in Fig. 9. Density is an essential structural property and one of the most important main properties of paper. Density correlates well with the number of contacts between fibers; hence density is strongly related to the porosity and strength of paper. As seen from Fig. 9, compared to water-formed paper at the same grammage level and wet pressing pressure, the foam-formed sheets had consistently higher density than water formed-paper. This was probably due to the more even formation of the foam-formed paper. 

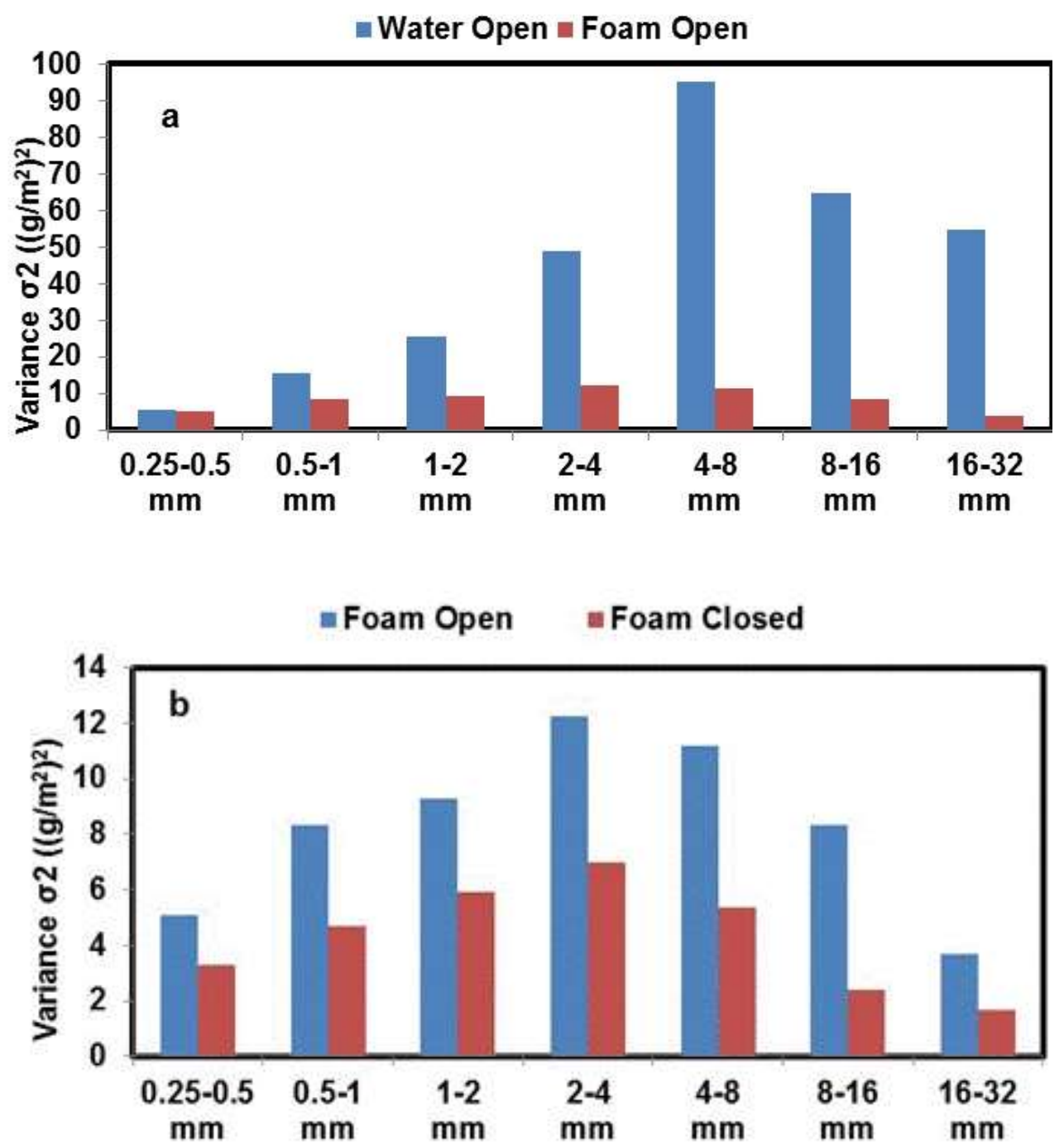

Fig. 8. Formation spectrum (variance) of the papers produced by water-laid and open foam-laid forming geometries (a). Formation spectrum with the small-scale variance of the papers produced using open and closed slice jet based forming geometries (b)

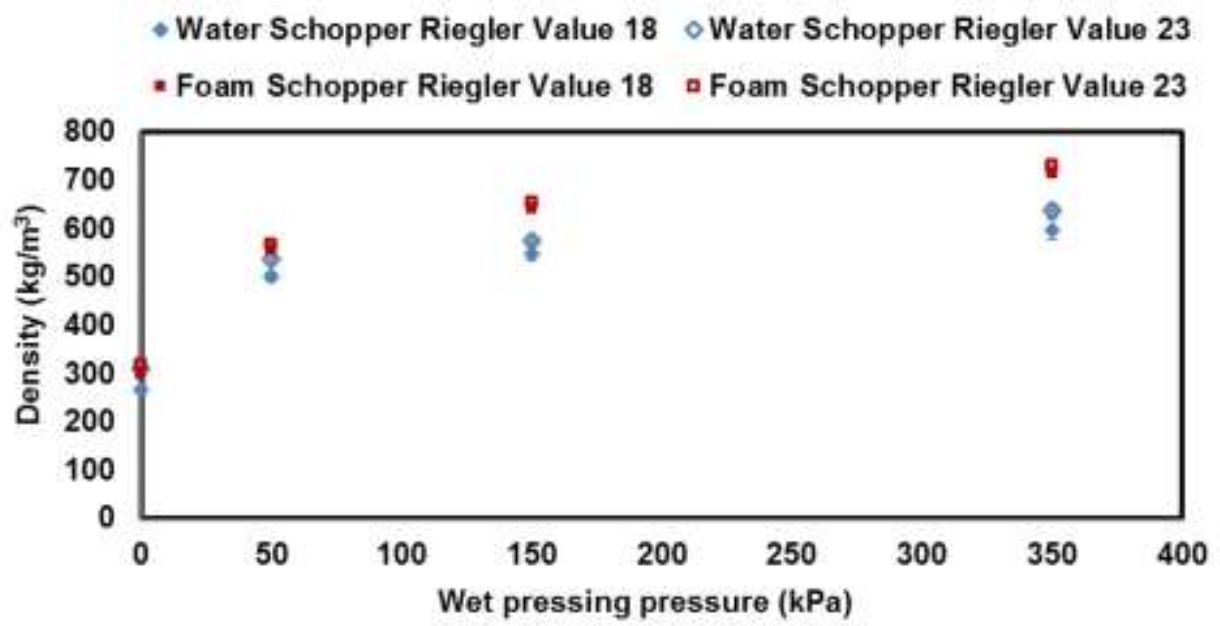

Fig. 9. Density of the dry sheets as a function of wet pressing pressure. Error bars show the standard deviation. 
Effect of jet-to-wire ratio on fiber orientation and formation

Tensile strength ratio was used to estimate the fiber orientation and $\beta$-formation to estimate the structural small-scale homogeneity of the web. The tensile strength ratio and specific $\beta$-formation as a function of jet-to-wire ratio are presented in Fig. 10. When varying the jet-to-wire ratio, the average flow rate into the headbox was kept constant at $7.2 \mathrm{l} / \mathrm{s} \pm 0.1 \mathrm{l} / \mathrm{s}$, and the forming speed was varied from 76 to $313 \mathrm{~m} / \mathrm{min}$ depending on the drag or rush conditions. The resulting papers had, on average, basis weight $80.2 \mathrm{~g} / \mathrm{m}^{2} \pm 4.2$ $\mathrm{g} / \mathrm{m}^{2}$, thickness $159.6 \mu \mathrm{m} \pm 10.1 \mu \mathrm{m}$, density $503.1 \mathrm{~kg} / \mathrm{m}^{3} \pm 11.3 \mathrm{~kg} / \mathrm{m}^{3}$, and bulk $2.0 \mathrm{~cm}^{3} / \mathrm{g}$ $\pm 0.1 \mathrm{~cm}^{3} / \mathrm{g}$. The lowest tensile strength ratio was achieved at a jet-to-wire speed difference of close to zero. A broad tensile strength ratio range from $\sim 3$ to $\sim 8$ was achieved for the closed headbox.

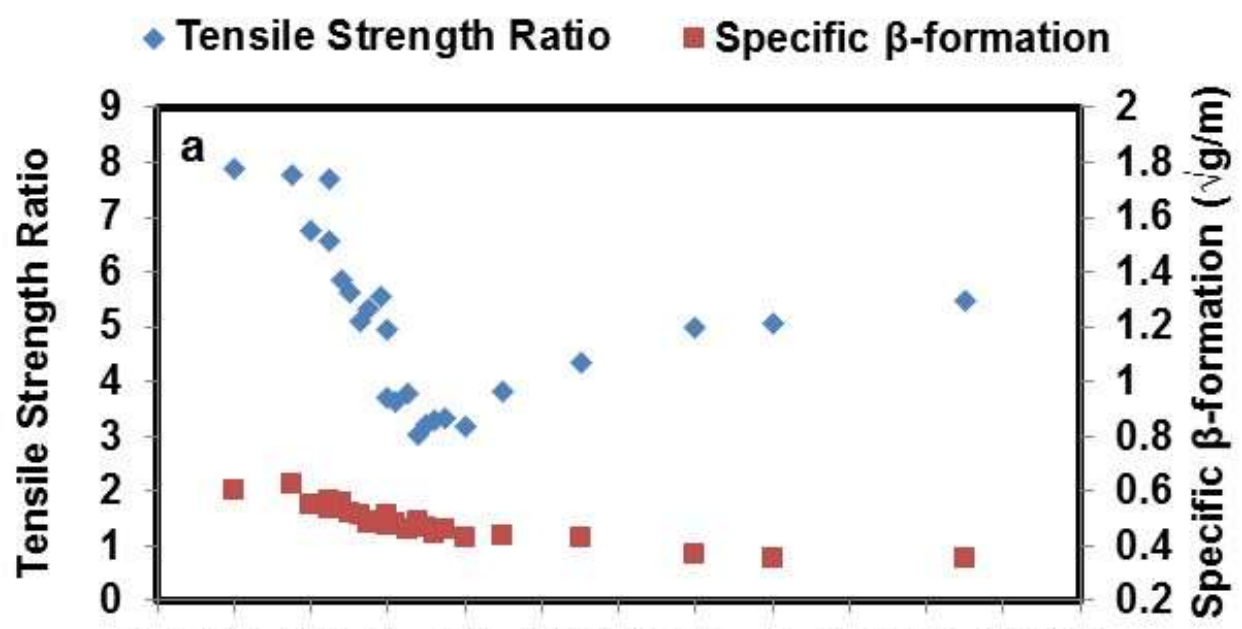
$\begin{array}{lllllllllllll}0.4 & 0.6 & 0.8 & 1 & 1.2 & 1.4 & 1.6 & 1.8 & 2 & 2.2 & 2.4 & 2.6 & 2.8\end{array}$
Jet-to-Wire Ratio

- Tensile Strength Ratio $\quad$ Specific $\beta$-formation

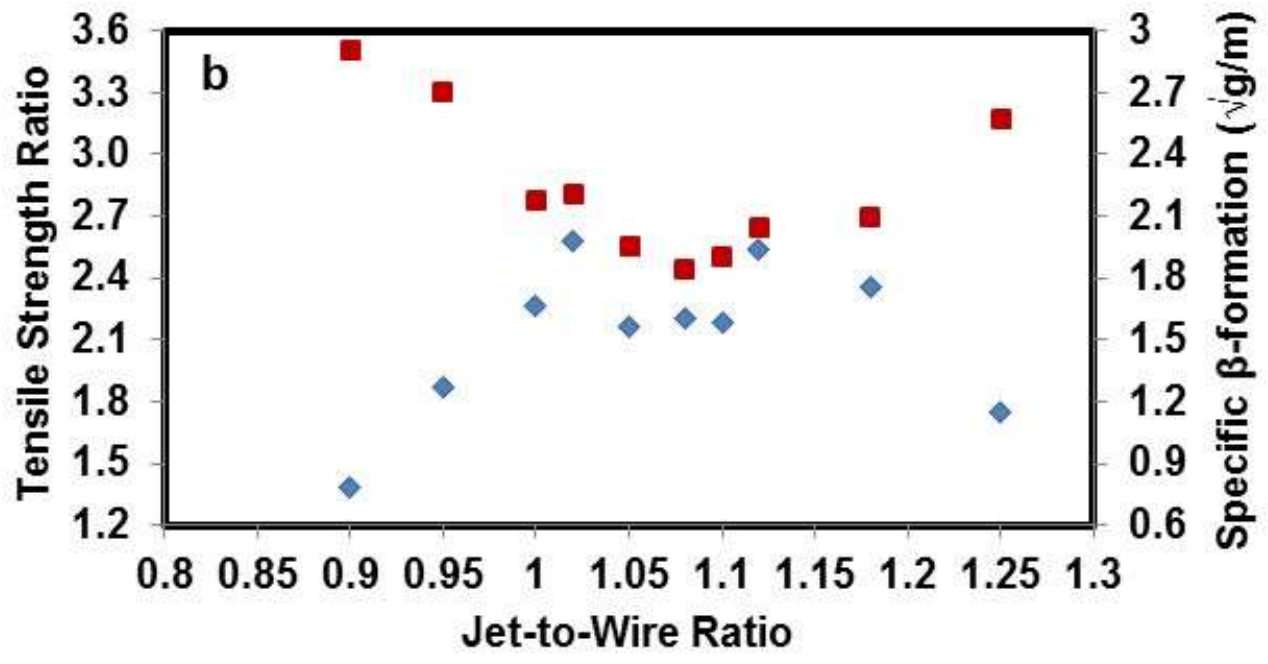

Fig. 10. Tensile strength ratio and specific $\beta$-formation (closed slice jet forming geometry) for foam-laid paper (a) and the corresponding curves (open slice jet forming geometry) for water-laid paper (b) as a function of the jet-to-wire ratio 
The maximum tensile strength ratio was run in drag conditions where the forming speed and the closed forming geometry enabled a highly oriented web structure at the same time. The achieved maximum tensile strength ratio was exceptionally high, at 8 . The corresponding behavior was not attained for the open slice jet based forming geometry, where the achieved maximum tensile ratio was around 3. One reason for this behavior is that the tapering closed forming geometry used orientated fibers more effectively in the machine direction at higher forming speed. The effect of change of shear was more notable in drag conditions than in rush conditions. When scaling-up foam-laid technology, the headbox and forming geometry has an essential role in web forming and in the foam removal phase.

Jet-to-wire series were used for determining optimal conditions for producing paper samples with a forming speed of $210 \mathrm{~m} / \mathrm{min}$ for both forming methods. Optimal forming conditions were determined such that the formation of the paper samples and the tensile strength ratio were at the appropriate level in both cases. This forming point was found to be a jet-to-wire ratio of 1.05 to 1.1 for water-laid paper, in line with our earlier study (Lehmonen et al. 2013) and also for foam-laid paper as shown in the present study (Fig. $10)$.

\section{Strength properties}

The role of surfactant that lowers surface tension is controversial. Various authors have examined the absorption of surfactants onto papermaking fibers, but their conclusions on the effects of paper properties are varied, in some cases an improvement in strength was indicated, whereas in others strength was lost (Nicolaysen and Borgin 1954; Touchette and Jennes 1960).

The mechanical properties of the paper samples are presented in Figs. 11 to 14. The mechanical strength properties are reported as a geometric mean value. In general, tensile strength is determined by the weakest point of the structure. The results from the samples, which were wet pressed and dried in the same way, showed an increase in tensile index as a function of density for both forming methods, foam having an even higher average tensile index with higher density levels (Fig. 11).

However, in the authors' earlier studies (Lehmonen et al. 2013), the average tensile index was somewhat higher for water-laid paper than for foam-laid paper as a function of density. The difference between the earlier foam results is probably due to the updated forming geometry and process configuration, which produced better formation of foamlaid paper (Fig. 6).

At the highest wet pressing level the foam-formed sheets had higher tensile strength compared to water-formed sheets. This suggests that wet pressing pressure has an overriding effect on surface tension forces in bringing fibers together and determining the sheet density and interfiber contacts (Lehmonen et al. 2019a).

Results at the same density level - considering the error bars - were roughly the same for strain at break and modulus of elasticity (Fig. 12 and 13). Due to improved formation, the same pressing level densified foam-formed papers more than water-formed papers. In general, these findings are similar to those reported by Niskanen and Kajanto (2008) with respect to the effect of wet pressing on modulus of elasticity. 


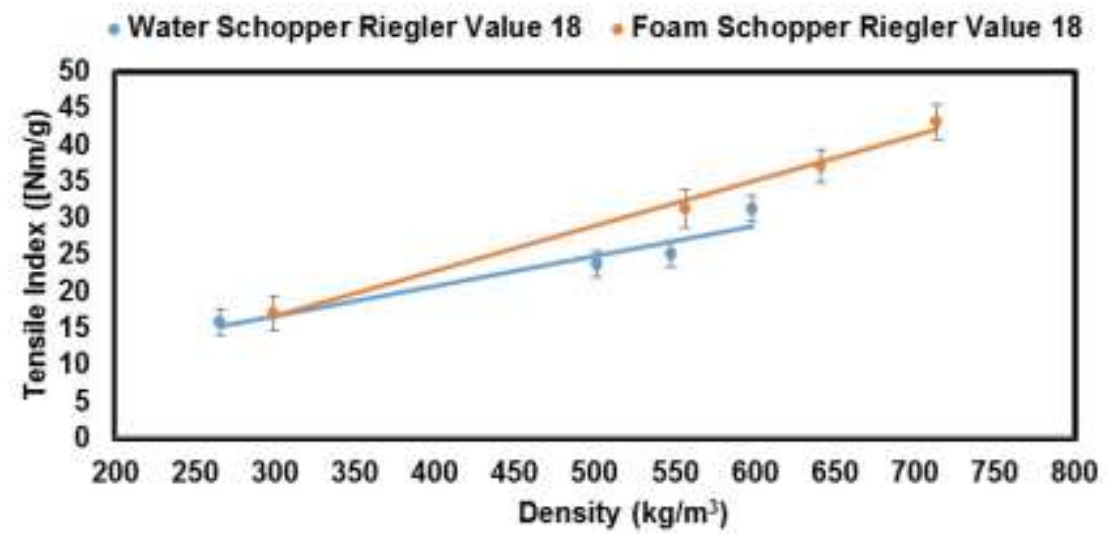

Fig. 11. Tensile strength of water-laid and foam-laid paper as a function of density. Error bars refer to standard deviation for 10 measurements. Error bars show the geometric mean standard deviation for 10 parallel measurements in both the MD and CD direction.

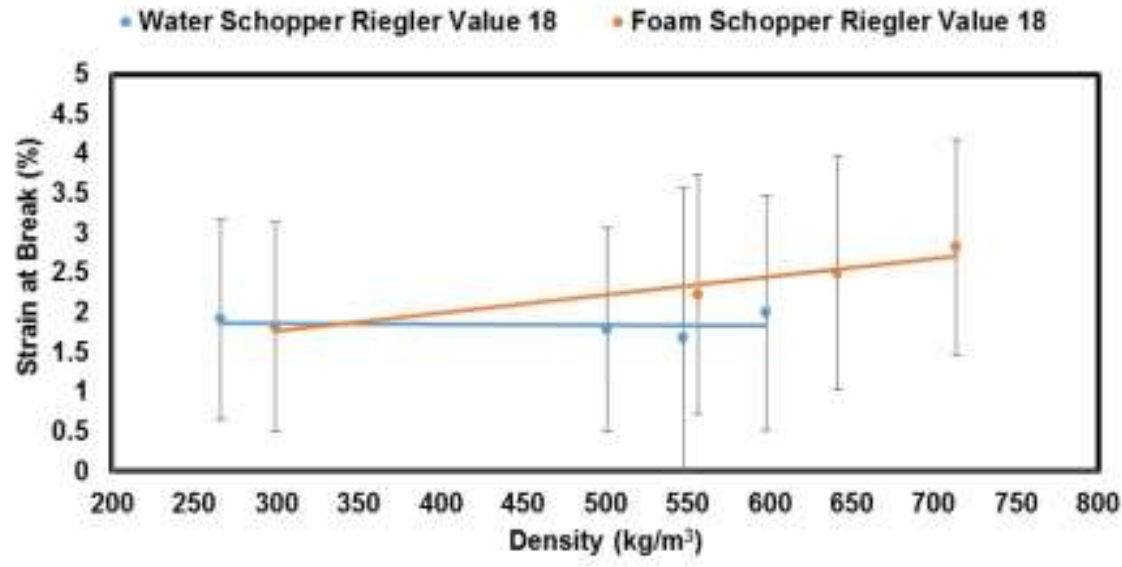

Fig. 12. Strain at break of water-laid and foam-laid paper as a function of density. Error bars show the geometric mean standard deviation for 10 parallel measurements in both the MD and CD direction.

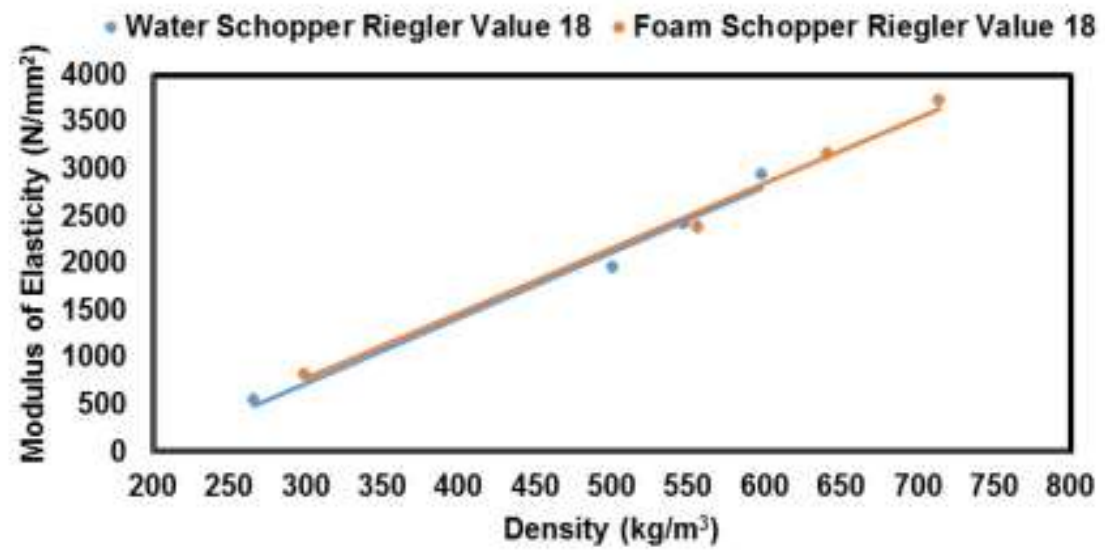

Fig. 13. Modulus of elasticity of water-laid and foam-laid paper as a function of density. Error bars show the geometric mean standard deviation for 10 parallel measurements in both the MD and CD direction. 
The z-directional strength results are shown in Fig. 14. The z-directional strength was lower for foam-laid papers as a function of density even though the quality of the foam-laid papers improved after the foam-laid process was updated. The higher average pore size of foam-laid papers (Kinnunen et al. 2013) has an effect on the z-directional strength (Lehmonen et al. 2013). Based on earlier studies, foam-formed samples have a remarkably high number of large pores compared to water-formed samples (Hjelt et al. 2011). These large pores act as weak points that initiate fractures and lower the $\mathrm{z}$ directional strength.

The formation of the sheet structure also affects z-directional strength. A felted sheet structure and fiber flocculation, i.e. poor formation, means that, in addition to fiber bonds, there are more z-directional fiber segments that carry the load (Niskanen and Kajanto 2008). Because the fibers are stronger than the bonds, the z-strength increases with decreasing formation uniformity.

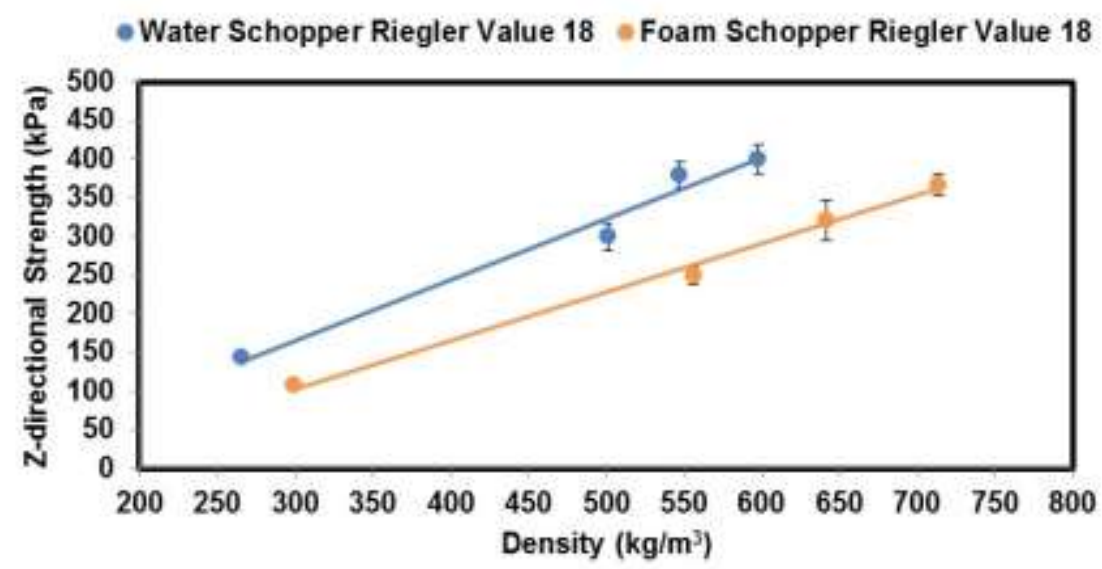

Fig. 14. Z-directional tensile strength of water-laid and foam-laid paper as a function of density. Error bars show the standard deviation for 10 parallel measurements.

Earlier studies have found that the presence of surfactants facilitates the removal of water from a structure and gives higher web dryness after the forming section (Touchette and Jennes 1960; Wiggins Teape 1973; Lehmonen et al. 2019a). In the present study, the dryness after the forming section was $22 \%$ for the lower refining level and correspondingly $21 \%$ for the higher refining level in the case of foam-laid paper. In the case of water-laid forming, average dryness was $18 \%$ for both refining levels. The dryness results indicated a higher average dryness for foam-laid webs after the forming section. One probable contributor to the higher dryness is the higher average pore size of foam-laid structures (Fig. 18).

\section{Bubble Size Distribution and Pore Size Distribution}

In addition to air content, bubble size also plays an important role in foam forming. The Sauter mean bubble size at different process stages is shown in Fig. 15. The Sauter mean bubble size of the process foam after the foam generator was $47 \mu \mathrm{m}$, and after the closed headbox $32 \mu \mathrm{m}$. The bubble size distribution after the open headbox and the closed headbox is shown in Fig. 16. The average bubble size was decreased after the modification of the foam-laid process research environment. The bubble volume is, however, dependent on the external pressure and temperature, and bubbles are prone to expand in the vacuum system. Bubbles also tend to coalesce and grow in size. However, bubbles can also be split 
into smaller bubbles under shear forces without a change in overall foam density. Fig. 15 shows the average Sauter mean radius $r[3,2]$ bubble size for different process locations. Observations during the trials confirmed that the size of process foam bubbles expands due to the change in vacuum level in the foam recovery phase. These changes in the process foam, i.e. changes in air content and bubble size, affect its viscosity and must be taken into account when sizing, for example, foam separators in the vacuum system. In Fig. 15, "Foam generator ingoing" is the foam drained from the forming section ("foamy water" in Fig. 1).

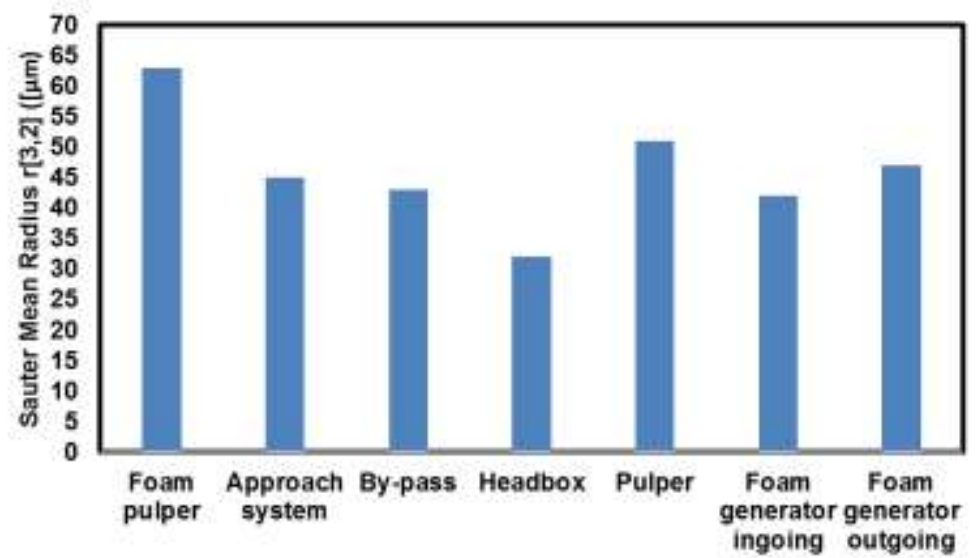

Fig. 15. Sauter mean radius $r[3,2]$ bubble size for different process locations

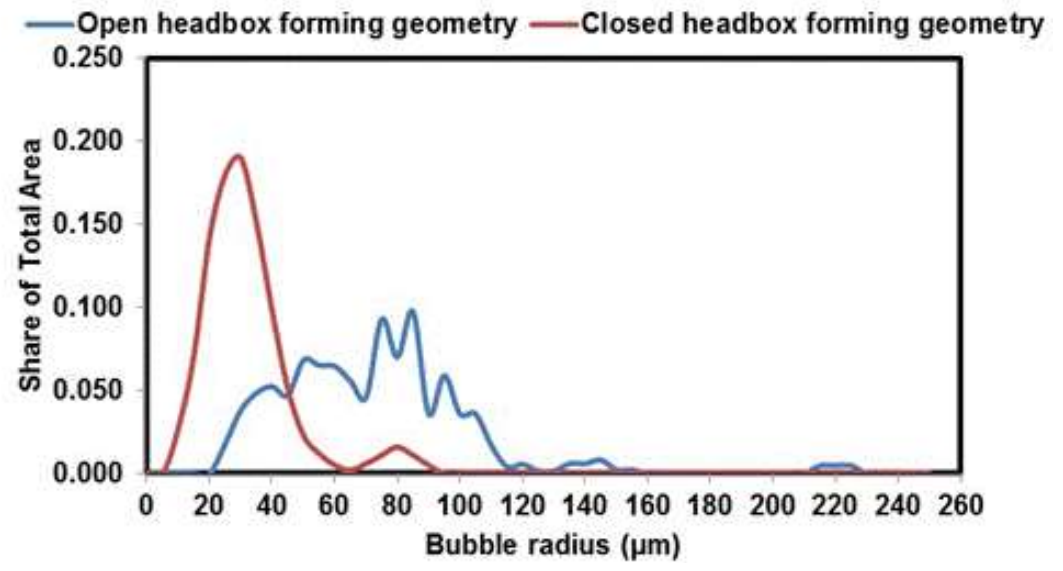

Fig. 16. Bubble size distribution after the open headbox forming geometry and closed headbox forming geometry

The pore size distributions of the water-laid and foam-laid samples are illustrated in Fig. 17. The mean pore size of the foam-laid and water-laid papers is shown in Fig. 18. The pore size distribution results are based on mercury porosimetry of the paper samples. In addition, an integrated area of pore size distribution is proportional to the total porosity. Foam-laid papers had higher porosity, and the higher amount of small pores compared to the water-laid sheets even at the highest wet pressing pressure, $350 \mathrm{kPa}$. However, the difference in mean pore size between water-laid and foam-laid samples diminished as a function of density. The modified pore size distribution might be a valuable feature for paper in some special paper grades, such as filter papers. 

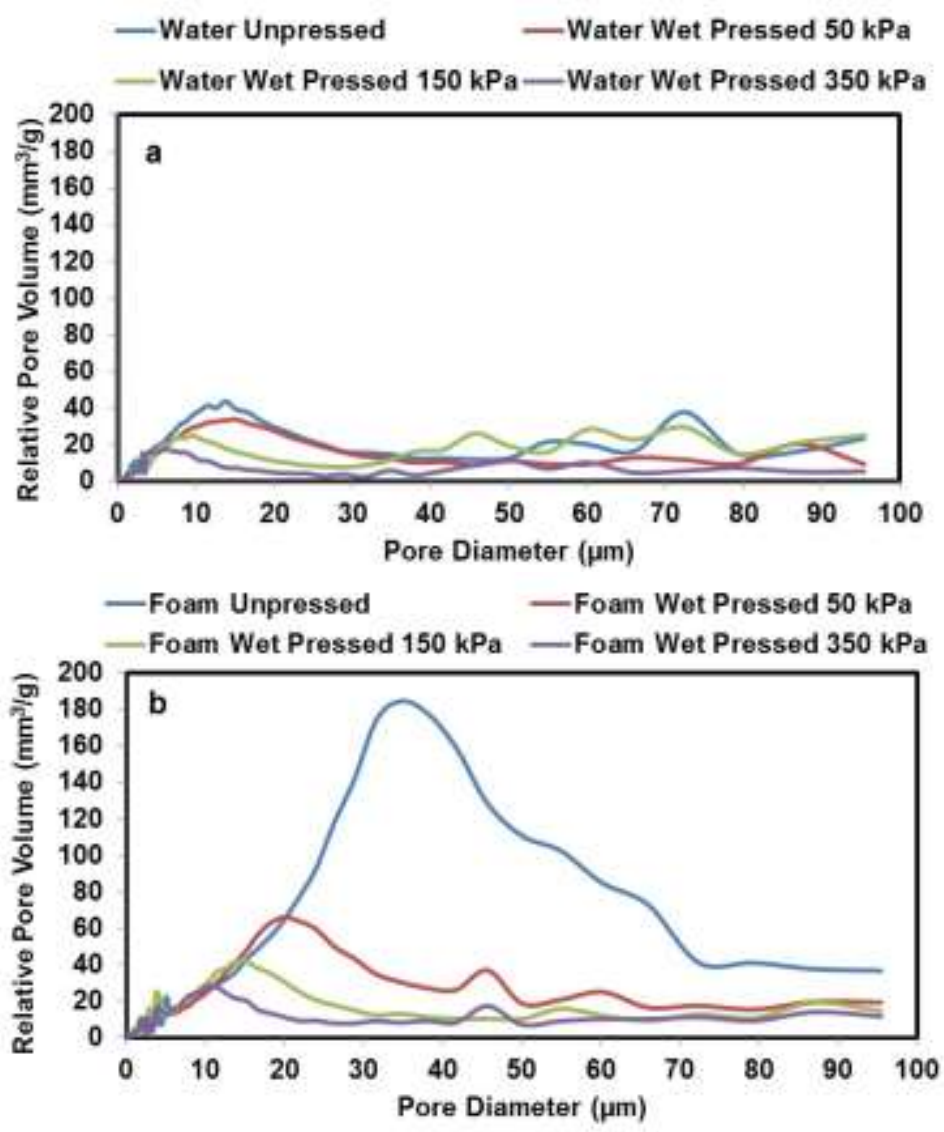

Fig. 17. Pore size distribution (non-normalized pore size distributions) for water-laid paper (a) and for foam-laid paper (b) as a function of pore diameter

- Water Schopper Riegler Value 18 - Foam Schopper Riegler Value 18

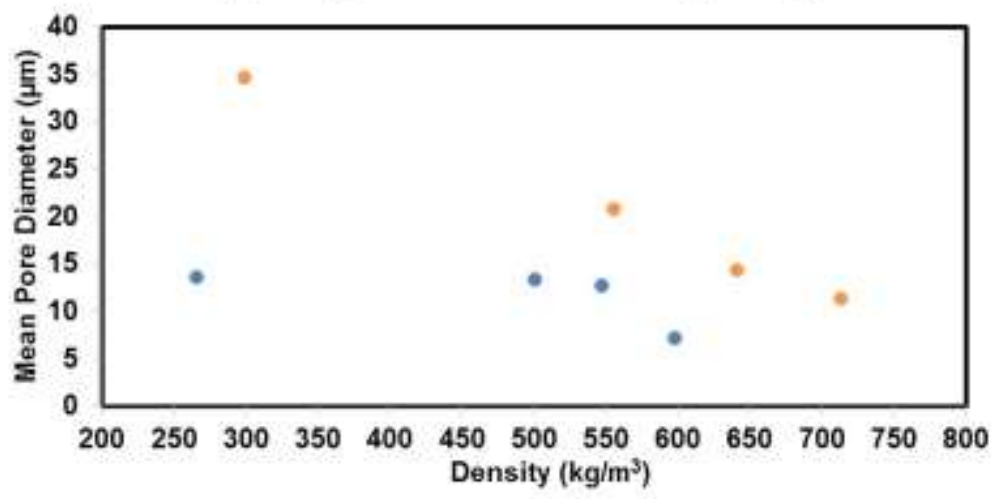

Fig. 18. Mean pore size for foam-laid and water-laid paper as function of density

Figure 19 shows the analysis of the x-ray microtomography images of water and foam-formed samples (Hjelt et al. 2011). The sphere color indicates the z-directional position of the sphere in the structure, with red being the top and blue the bottom. In the foam-formed sample in the figure, big pores construct channels through the structure in the z-direction, whereas in the water-formed sample, large pores are almost isolated. The channels in the foam-formed samples are very beneficial for drainage properties. 


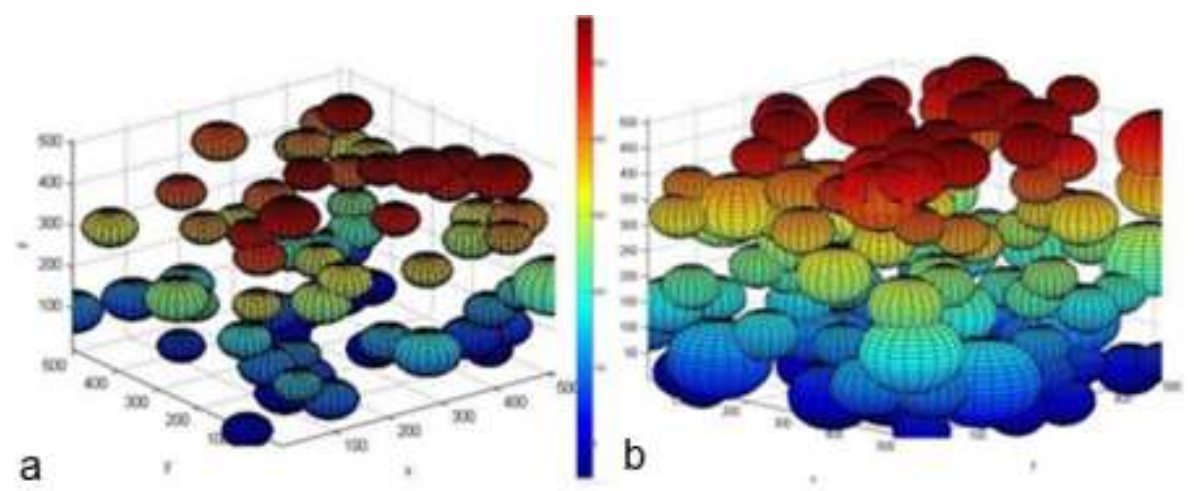

Fig. 19. Characterization of the pore network by balls of radius larger than $52 \mu \mathrm{m}$. a) Waterformed sample and b) foam-formed sample. Red indicates pores at the top of the paper, blue indicates pores at the bottom (Hjelt et al. 2011 (copyright permission)).

\section{Effect of Refining}

Strength properties

The mechanical strength properties of chemical softwood pulps are presented in Figs. 20 to 22. According to the present findings, the refining level of chemical softwood pulp could be reduced without losing in-plane tensile strength properties or reducing the formation of foam-laid paper. The average tensile strength properties of foam-laid paper as a function of density are comparable with water-laid paper, as shown in Fig. 19.

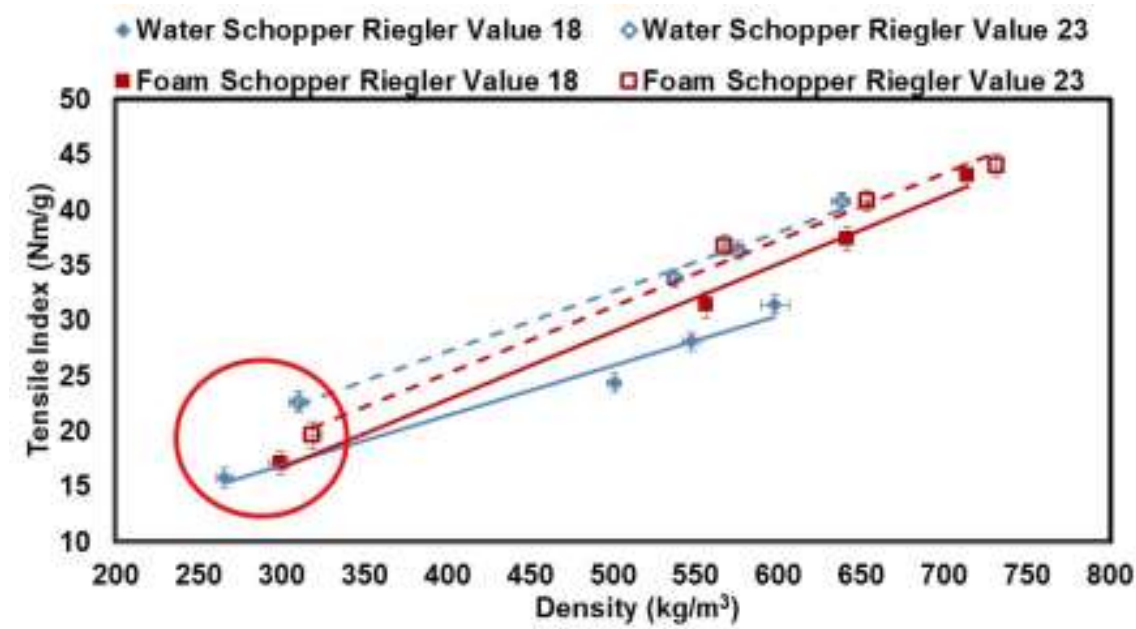

Fig. 20. Tensile strength of water-laid and foam-laid paper as a function of density. Error bars show the $95 \%$ confidence level.

Refining slightly improved the tensile strength of foam-laid paper, but not statistically significantly ( $95 \%$ confidence level). The influence of refining was remarkably stronger on water-laid paper. This is particularly evident with the unpressed samples in Fig. 20, where the difference between water-formed samples at the different refining levels is clearly statistically noticeable. For the unpressed foam-formed samples, the difference introduced by refining is much smaller and within statistical error. Similar trends are also observed for the wet pressed samples, but the statistical significance of the differences are weaker. According to these strength results, it would be possible to reduce the amount of refining without significantly weakening the strength properties of foam-laid paper. In 
addition, the results suggest that the possible presence of the used surface active agent in the samples does not influence the strength properties.

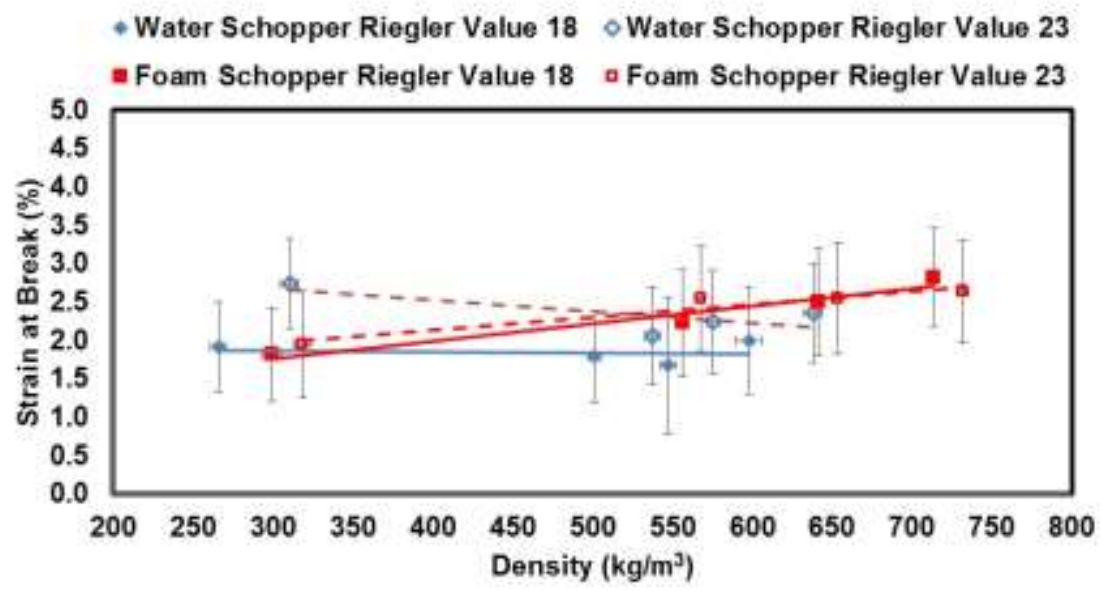

Fig. 21. Strain at break of water-laid and foam-laid paper as a function of density. Error bars show the $95 \%$ confidence level.

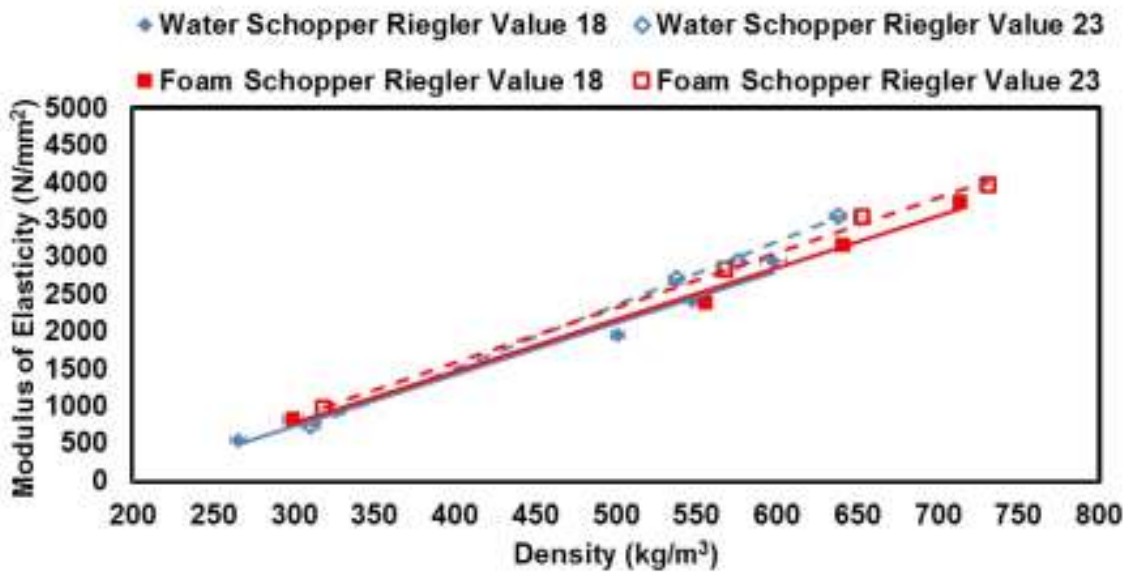

Fig. 22. Modulus of elasticity of water-laid and foam-laid paper as a function of density. Error bars show the $95 \%$ confidence level.

\section{Short Column Compression Test}

The short column compression test (SCT) measurement predicts the end-use characteristics of carton boxes, i.e. how they stack on top of each other. The short column compression test was measured in the machine direction. It should be remembered that the ${ }^{\circ}$ SR 18 foam-formed samples had higher fiber orientation. The present study found that the SCT values were roughly at the same level at the higher refining level (the slope of the curves was roughly the same at the higher refining level) (Fig. 23). An advantage of the studied foam forming technology was observed at the lowest density point of the lower refining level. The fibers are stiffer at the lower refining level (Abitz and Luner 1989). Despite the limited number of measurements, and taking into account the differences in fiber orientation, the result indicated that the used foam forming technology makes it possible to utilize the properties of stiffer fibers at low sheet density without weakening web formation, leading to improvements in the properties of the fiber network. 

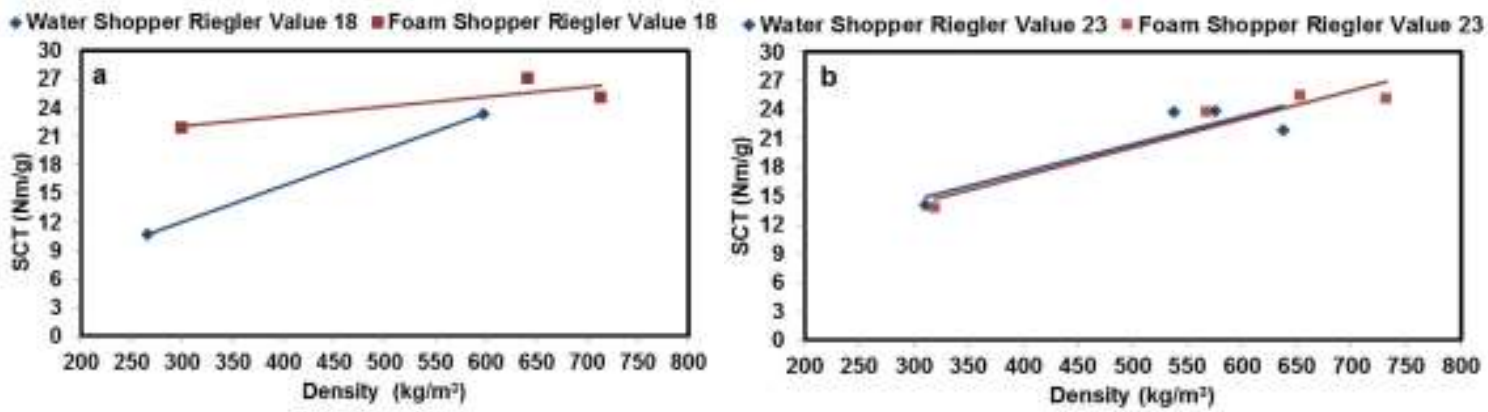

Fig. 23. Short span compression test (SCT) of water-laid and foam-laid paper for ${ }^{\circ}$ Riegler value 18 (a) and Shopper Riegler value 23 (b) as a function of density. Error bars show the standard deviation for 10 parallel measurements.

The mechanical properties of a sheet of paper can be described and analyzed by estimating the bonded and unbonded area of fibers in the sheet. This can be done optically by measuring the light scattering properties of the sheet (Haselton 1953, 1954). This method assumes that the fiber surfaces that are in optical contact, and thereby do not scatter light, are bonded, and that the change in the light scattering coefficient is due to the change in the bonded area. In the present study, the scattering coefficient results indicated that at the same density level the foam-laid structures are less bonded (Fig. 24). However, in foamlaid structures generally, higher density levels were achieved when the same wet pressing conditions were applied. The higher density levels were probably due to the improved web formation. The higher density level enhances the tendency of the fiber network to form bonds. Considering the overall picture of water-laid and foam-laid structures, the scattering coefficient results indicated fewer bonds in the foam-laid structures. This difference may be due to the effect of the surfactant used on the surface tension and on the strength development. A part of the effect may be also due to the effect of bubbles on the paper structure (Lehmonen et al. 2019a).
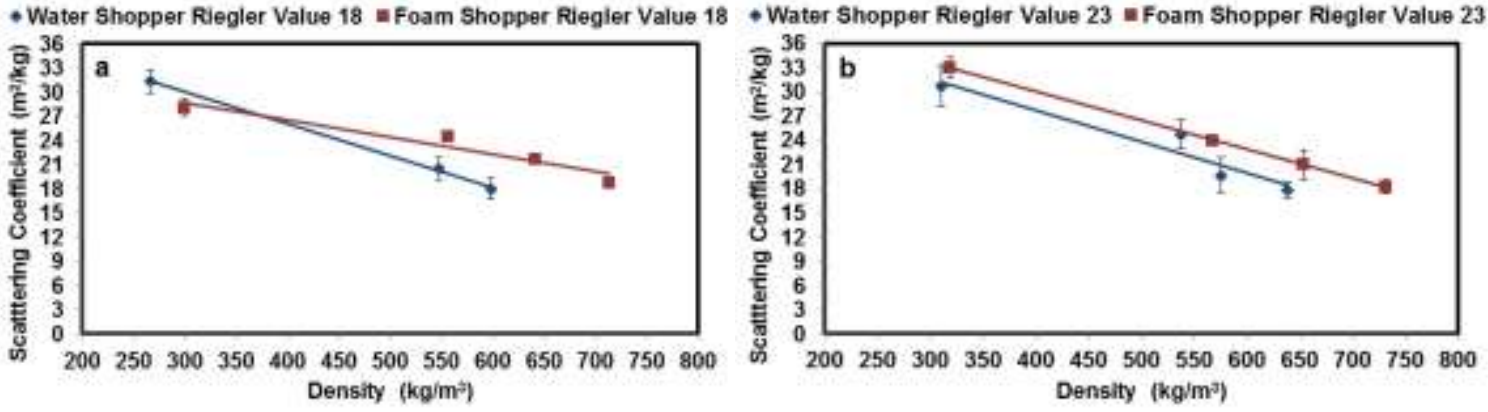

Fig. 24. Scattering coefficient for Shopper Riegler value 18 (a) and Shopper Riegler value 23 (b) as a function of density. Error bars show the standard deviation for 10 parallel measurements.

\section{CONCLUSIONS}

1. In this study, two process configurations were compared for foam. The studies were carried out using a water-laid former and the same environment modified for foam forming. The open slice-jet forming geometry was further modified and replaced with a closed headbox and an additional foam pulper in order to increase forming speed, improve dewatering, and to attain better foam stability and foam recovery in the 
forming section. The process conditions and formed web properties can be affected by headbox and former solutions, and therefore the major change was related to the forming geometry. Formation and formation spectra were better with foam forming than with water forming. In foam forming, the formation and formation spectra with the closed forming geometry were even better in all wavelengths than with the open forming geometry.

2. The strength values of foam-formed samples were comparable with water-formed sheets, often better. Z-directional strength was, however, worse due to better formation (z-directional strength increases with decreasing formation) and lower interfiber bond area, as suggested based on the light scattering values.

3. Refining of chemical softwood pulp increased the average tensile strength of waterlaid and foam-laid samples as a function of density. The influence of refining was remarkably stronger on water-laid paper. In the case of densified structures, i.e. wet pressed samples, the strength was at the same level when the refined chemical softwood pulps were used in both forming technologies. The strength of the unrefined foamformed samples was only slightly weaker, whereas the water-formed samples were markedly weaker. The reason for the foam-formed sample's strength is better formation. According to these strength results, it is possible to reduce the amount of refining without weakening the strength properties of foam-laid paper. This result allows cost savings in the refining phase of fibers as a lower level of refining could be used.

4. Foam-laid papers had higher porosity and the higher amount of small pores compared to the water-laid sheets even at the highest wet pressing pressure, $350 \mathrm{kPa}$. However, the difference in mean pore size between water-laid and foam-laid samples diminished as a function of density. The ability to modify pore size distribution is a beneficial feature for some special paper grades, such as filter paper.

5. The higher viscosity of the foam compared to water and the closed forming geometry used orientated the fibers more effectively in the machine direction at higher forming speed, resulting in higher fiber orientation in the foam-formed sheets and also the ability to control the MD/CD-strength ratio over a wide range (3...8) without deteriorating the uniformity of formation. This also suggests that very high MD strength values are achievable.

6. The improvements made to the process, especially mixing conditions and forming geometry, led to a more stable foam process, as evident, e.g., from the improved formation of the paper samples.

\section{ACKNOWLEDGMENTS}

The research leading to these results was performed as part of the "Efficient Networking towards Novel Products and Processes" (EffNet) research program of Forestcluster Ltd. The EffNet program has received funding from Tekes - the Finnish Funding Agency for Technology and Innovation (nowadays Business Finland), and Forestcluster Ltd. 


\section{REFERENCES CITED}

Abitz, P., and Luner, P. (1989). "The effect of refining on wet fiber flexibility and its relationship to sheet properties," in: Fundamentals of Papermaking Fundamental Research Symposium held at Cambridge, C. F. Baker and V. W. Punton (ed.), Mechanical Engineering Publications Ltd. London. pp. 67.

Al-Qararah, A. M., Ekman, A., Hjelt, T., Ketoja, J. A., Kiiskinen, H., Koponen, A., and Timonen, J. (2015). "A unique microstructure of the fiber networks deposited from foam-fiber suspensions," Colloids and Surfaces A: Physicochem. Eng. Aspects 482, 544-553. DOI: 10.1016/j.colsurfa.2015.07.010

Engelsen, C. W., Isarin, J. C., Gooijer, H., Warmoeskerken, M., and Wassink, J. G. (2002). "Bubble size distribution of foam," AUTEX Res. J. (2)1, 14-27.

Hanson, J. P. (1977). "What's going on in non-wovens," Pulp \& Paper 51(11), 97-102.

Haselton, W. R. (1954). "Gas adsorption by wood, pulp and paper 1 . The low temperature adsorption of nitrogen, butane and carbon dioxide by sprucewood and its components," Tappi 37(9), 404-412.

Haselton, W. R. (1955). "Gas adsorption by wood, pulp and paper 2. The application of gas adsorption techniques to the study of the area and structure of pulps and the unbonded and bonded area of paper," Tappi 38(12), 716-723.

Hjelt, T., Kinnunen K., Lehmonen J., Beletski N., Hellén E., Liljeström V., Serimaa R., Miettinen A., and Kataja M. (2011). "Intriguing structural and strength behaviour in foam forming," Progress in Paper Physics Seminar Graz, Austria.

Htun, M., and Fellers C. (1982). "The invariant mechanical properties of oriented handsheets," Tappi 65(4), 113-117.

Junker, B. (2006). "Measurement of bubble and pellet size distributions: Past and current image analysis technology," Bioprocess Biosyst Eng. 29(3), 185-206. DOI: 10.1007/s00449-006-0070-3

ISO 5267-1. (1998). "Pulps - Determination of drainability - Part 1: Shopper Riegler Method," International Organization for Standardization, Geneva, Switzerland.

ISO 5269:1. (2005). "Pulp - Preparation of laboratory sheets for physical testing - Part 1: Conventional sheet-former method," International Organization for Standardization, Geneva, Switzerland.

ISO 536. (1995). "Paper and Board - Determination of grammage," International Organization for Standardization, Geneva, Switzerland.

ISO 534. (2005). "Paper and Board - Determination of thickness and apparent bulk density or apparent sheet density," International Organization for Standardization, Geneva, Switzerland.

ISO 5270. (2012). "Pulps - Laboratory sheets - Determination of physical properties," International Organization for Standardization, Geneva, Switzerland.

ISO 15754. (2009). "Paper and Board - Determination of Z-directional tensile strength," International Organization for Standardization, Geneva, Switzerland.

ISO 9416. (2009). "Determination of light scattering and absorption coefficients (using Kubelka-Munk theory)," International Organization for Standardization, Geneva, Switzerland.

Kerekes, R. J., Soszynski R. M., and Tam Doo P. A. (1985). "The flocculation of pulp fibers," in: The $8^{\text {th }}$ Fundamental Research Symposium, 15-20 September, Oxford, pp. 265-310. 
Kerekes, R. J. (2006). "Rheology of fibre suspensions in papermaking: An overview of recent research," Nord. Pulp Paper Res. J. 21(5), 598-612.

Kidner, T. L.W. (1974). "The radfoam process for fine papers," Wiggins Teape Research and Development Ltd. Paper Technology. pp. 346-351.

Kinnunen-Raudaskoski, K. (2017). Foam as a Carrier Phase-A Multipurpose Technology for Industrial Applications, Ph.D. Dissertation, Aalto University, Espoo, Finland.

Kinnunen, K., and Hjelt, T. (2016). "Fibrous web of paper or board and method of making the same," U.S. Patent No. 9334610.

Kinnunen, K., and Hjelt, T. (2017). "Method of producing boards," F.I. Patent No. 126699.

Kinnunen, K., Lehmonen, J., Beletski, N., Jetsu, P., and Hjelt, T. (2013). "Benefits of foam forming technology and its applicability in high NFC addition structures," in: $15^{\text {th }}$ Fundamental Research Symposium, 8-11 September, Oxford, UK. Proceedings of the 15th Pulp and Paper Fundamental Research Symposium, Cambridge.

Koivula, H., Toivakka M., and Gane P. (2011). "Short time spreading and wetting of offset printing liquids on model calcium carbonate coating structures," Journal of Colloid and Interface Science 369(2012), 426-434. DOI: 10.1016/j.jcis.2011.11.065

Koponen, A., Torvinen, K., Jäsberg, A., and Kiiskinen, H. (2016). "Foam forming of long fibers," Nord. Pulp Pap. Res J. 31(2), 239-247. DOI: 10.3183/npprj-2016-31-02p239-247

Kruglyakov, E. (1999). Foam and Foam Films Theory, Experiment and Application, Amsterdam, the Netherlands, pp. 586 and 719-721.

Lappalainen, T., and Lehmonen J. (2012). "Determinations of bubble size distribution of foam-fibre mixture using circular hough transform," Nord. Pulp Paper Res. J. 27(5), 930-939. DOI: 10.3183/NPPRJ-2012-27-05-p930-939

Lehmonen, J., Jetsu, P., Kinnunen, K., and Hjelt, T. (2013). "Potential of foam-laid forming technology in paper applications," Nord. Pulp Pap. Res J. 28(3), 392-398. DOI: 10.3183/npprj-2013-28-03-p392-398

Lehmonen, J., Retulainen, E., Kinnunen-Raudaskoski, K., Paltakari, J., and Koponen, A. (2019a). "Dewatering of foam-laid structures and formed web properties," Cellulose Published online: 16 November 2019. Correction to: Dewatering of foam-laid and water-laid structures and the formed web properties Published online: 21 December 2019. Original article DOI: 10.1007/s10570-019-02842-x and Correction DOI: 10.1007/s10570-019-02913-z

Lehmonen, J., Rantanen T., and Kinnunen-Raudaskoski K. (2019b). "Upscaling of foam forming technology for pilot scale," Tappi Journal 18(8), 461-471.

Martinez, D. M., Buckley, K., Jivan, S., Lindström, A., Thiruvengadaswamy, R., Olson, J. A., Ruth, T. J., and Kerekes, R. J. (2001). "Characterizing the mobility of papermaking fibres during sedimentation," in: The $12^{\text {th }}$ Fundamental Research Symposium, Oxford, UK, pp. 225-254.

Moruzzi, R. B., and Reali M. A. P. (2010). "Characterization of micro-bubble size distribution and flow configuration in DAF contact zone by a non-intrusive image analysis system and tracer tests," Water Sci. Techn. 61(1), 253-262. DOI: 10.2166/wst.2010.784

Nicolaysen, V. B., and Borgin, K. (1954). "The effect of surface active agents on purified cellulose fibers," Norsk Skogindustrie 8(54), 260-267. 
Niskanen K., and Kajanto I. (2008). "Paper structure," and "Structural mechanics of paper and board," in: Paper Making Science and Technology, Book 16: Paper Physics. Finnish Paper Engineers' Association. Helsinki, Finland, pp. 11-58, 229-264.

Phongikaroon, S., Herrmann, S. D., Li, S. X., and Simpson, M. F. (2006). "Measurement and analysis of gas bubbles near a reference electrode in aqueous solutions," Ind. Eng. Chem. Res. 45(22), 7679-7687. DOI: 10.1021/ie0606643

Punton, V. W. (1975). "The use of an aqueous foam as a fibre-suspending medium in quality papermaking," in: Proceedings of a Symposium Organized by the Society of Chemical Industry, London.

Radvan, B., and Gatward, A. P. J. (1972). "The formation of wet-laid webs by a foaming process," Tappi 55(5), 748-751.

Riddell, M. C., and Jenkins, B. (1976). "The use of non-surfactant foaming agents in the paper machine," Paper Technology and Industry April, pp. 80-83.

Sauter, J. (1926). Die Grössenbestimmung der im Gemischnebeln von Verbrennungskraftmaschinen vorhandenen Brennstoffteilchen, VDI-Verl., Berlin, Germany.

Smith, M. K., and Punton V. W. (1975). "Foam can improve formation," Pulp \& Paper Canada 76(1), 55-58.

Smith, M. K., Punton V. W., and Rixson A. G. (1974). "The structure and properties of paper formed by a foaming process," Tappi 57(1), 107-111.

Touchette, R. V., and Jennes, L. C. (1960). "Effect of surface active agents on drainage and physical strength of sulfite pulp," Tappi 43(5), 484-489.

Weaire, D., and Hutzler, S. (1999). The Physics of Foams, Oxford University Press, UK.

Wiggins, T. (1973). "Make paper with foam not without it. A report from Wiggins Teape Research and Development Ltd," Pulp Trade Journal 16, 28-31.

Wiggins, T. (1971). "New process uses foam in papermaking instead of avoiding it," Paper Trade Journal 29, 44-45.

Article submitted: February 19, 2020; Peer review completed: May 24, 2020; Revised version received and accepted: June 25, 2020; Published: July 2, 2020.

DOI: 10.15376/biores.15.3.6309-6331 